\title{
Analytical Solution of The Two-Qubit Quantum Rabi Model
}

\author{
D. A. M. Abo-Kahla ${ }^{1, *}$, M. Abdel-Aty ${ }^{2}$ and Salah A. Aly Ahmed ${ }^{3}$ \\ ${ }^{1}$ Department of Mathematics, Faculty of Education, Ain Shams University, Cairo, Egypt \\ ${ }^{2}$ University of Science and technology, Zewail City of Science and Technology, Giza, Egypt \\ ${ }^{3}$ Department of Computer Sciences, Faculty of Science, Fayoum University, Egypt \\ * corresponding author: doaa_abukahla@ymail.com
}

\begin{abstract}
In this paper, an analytical solution of the two-qubit Rabi model for the general case is presented. Furthermore, a comparison between the information entropies and the Von Neumann entropy $S\left(\rho_{A}\right)$ is given for some special values of the qubit-photon coupling constants for the two qubits and the detuning parameters. It is demonstrated that oscillations of the occupation probabilities $\rho_{11}, \rho_{22}, \rho_{33}$ and $\rho_{44}$ are equivalent to the case of the spontaneous emission. The occupation probability $\rho_{11}$ reaches the case of sudden death, when the detuning parameter $\Delta_{2}$ equals zero.
\end{abstract}

Keywords- Quantum Optics and Rabi Model.

\section{INTRODUCTION}

The light-matter interaction is one of the fundamental problems investigated in many aspects of modern physics ranging from quantum optics to quantum information processing and to condensed matter physics. The simple description of quantum light-matter interaction, namely the interaction of a qubit with a harmonic oscillator, is given by Rabi model [1]. This simple model has been widely used in atomic physics, optical physics and condensed matter physics [2, 3]. In addition, it has been experimentally tested in different physical systems, such as cavity systems [4], trapped ions [5], quantum dots [6], superconducting circuits [7-12] and photonic superlattices [13,14]. Finding solutions of the quantum optical Hamiltonian has been an important topic in the existing literature [15-19]. Rabi model in the symmetric case has been studied by using Fulton-Gouterman transformation [20], in which solutions of this model are proposed as a function of energy [21-28]. Most of these solutions are concentrated the model calculations mainly on the $G$ function. However, other attempts considered Rabi model solution as a function of time. However, such attempts used either rotating wave approximation (RWA), which leads to JaynesCummings model [29, 30], or generalized rotatingwave approximation (GRWA) of only one qubit [31]. In this work, we focus on the analytical solution of the two-qubit Rabi model for the general case. By calculating the occupation probabilities of the the multi-qubit
Rabi model, we show that it is possible to generate oscillations to a great extent look like the case of the spontaneous emission in different situations. In section [II we describe the Hamiltonian of the given system, and obtain the explicit analytical solution of the model describing the dynamics of multi-qubit Rabi model. In Section [III we discuss the Von Neumann entropy $S\left(\rho_{A}\right)$ and the information entropies $H\left(\sigma_{Z}\right)$, $H\left(\sigma_{Y}\right)$ and $H\left(\sigma_{X}\right)$ by changing the qubit-photon coupling constants for the two qubits and the detuning parameters.

\section{Exact Solution of The Model}

We briefly discuss the general formalism to characterize the dynamics of the multi-qubit Rabi model for the general case, for more details, see [32]. The Hamiltonian of the system can be written as $(\hbar=1)$ :

$$
H=\omega a^{\dagger} a+\left(a^{\dagger}+a\right) \sum_{j=1}^{2}\left(\mu_{j} \sigma_{j, x}+\delta_{j} \sigma_{j, z}\right)
$$

where $a^{\dagger}$ and $a$ are the single-mode photon creation and annihilation operators with frequency $\omega$, respectively. $\sigma_{i x}, \sigma_{i z}(i=1,2)$ are the Pauli matrices of the $i^{\text {th }}$ qubit. $\delta_{j}$ is the detuning parameter of the qubit $j$, and $\mu_{j}$ is the qubit-photon coupling constants.

Here we start with the two-qubit case and try to 
find an analytic solution

$$
\begin{aligned}
H= & \omega a^{\dagger} a+\mu_{1} \sigma_{1 x}\left(a^{\dagger}+a\right)+\mu_{2} \sigma_{2 x}\left(a^{\dagger}+a\right) \\
& +\delta_{1} \sigma_{1 z}+\delta_{2} \sigma_{2 z} \\
& =\omega\left\{a^{\dagger} a+g_{1} \sigma_{1 x} I_{2}\left(a^{\dagger}+a\right)+g_{2} I_{1} \sigma_{2 x}\left(a^{\dagger}+a\right)\right. \\
& \left.+\Delta_{1} \sigma_{1 z} I_{2}+\Delta_{2} I_{1} \sigma_{2 z}\right\}
\end{aligned}
$$

where $g_{1}=\frac{\mu_{1}}{\omega}, g_{2}=\frac{\mu_{2}}{\omega}, \Delta_{1}=\frac{\delta_{1}}{\omega}$ and $\Delta_{2}=\frac{\delta_{2}}{\omega}$. One may consider the following unitary transformations $S_{1}=\frac{1}{\sqrt{2}}\left(\sigma_{1 x}+\sigma_{1 z}\right)$ and $S_{2}=\frac{1}{\sqrt{2}}\left(\sigma_{2 x}+\sigma_{2 z}\right)$ to $H$ to obtain $\grave{H}$ as

$$
\begin{aligned}
\grave{H}= & \omega\left\{a^{\dagger} a+g_{1} \sigma_{1 z}\left(a^{\dagger}+a\right)+g_{2} \sigma_{2 z}\left(a^{\dagger}+a\right)\right. \\
& \left.+\Delta_{1} \sigma_{1 x}+\Delta_{2} \sigma_{2 x}\right\}
\end{aligned}
$$

where $\grave{H}=U^{\dagger} H U$ and $U=S_{2} \otimes S_{1}=$ $\frac{1}{2}\left(\begin{array}{cccc}1 & 1 & 1 & 1 \\ 1 & -1 & 1 & -1 \\ 1 & 1 & -1 & -1 \\ 1 & -1 & -1 & 1\end{array}\right)$, with $S_{i}=\frac{1}{\sqrt{2}}\left(\begin{array}{cc}1 & 1 \\ 1 & -1\end{array}\right)$. Then, the general solution of the eigen value problem can be written as follows:

$$
\begin{aligned}
|\grave{\Psi}\rangle= & \sum_{k=1}^{4} \exp \left(-i \omega_{k} t\right)\left|\grave{\Psi}_{k}\right\rangle \\
& =\sum_{k=1}^{4} \exp \left(-i \lambda_{k} t\right)\left(\begin{array}{c}
Q_{1 k} \\
Q_{2 k} \\
Q_{3 k} \\
Q_{4 k}
\end{array}\right)|\alpha\rangle,
\end{aligned}
$$

where

$$
\begin{aligned}
& \lambda_{k}=|\alpha|^{2}+(-1)^{k} x_{3}, \\
& Q_{1 k}=\frac{y_{1} y_{2} \zeta_{1}+(-1)^{k} \zeta_{2}-x_{1} x_{3}}{2 \Delta_{1} \Delta_{2} x_{3}}, \\
& Q_{2 k}=(-1)^{k}\left(\frac{y_{1}\left[y_{1}+(-1)^{k} x_{3}\right]+\Delta_{1}^{2}+(-1)^{\frac{k(k+1)}{2}} x_{1}}{2 \Delta_{1} x_{3}}\right), \\
& Q_{3 k}=(-1)^{k}\left(\frac{y_{2}\left[y_{2}+(-1)^{k} x_{3}\right]+\Delta_{2}^{2}+(-1)^{\frac{k(k+1)}{2}} x_{1}}{2 \Delta_{2} x_{3}}\right), \\
& Q_{4 k}=\frac{1}{2}, y_{1}=(g+\grave{g}) \operatorname{Re}(\alpha), y_{2}=(g-\grave{g}) \operatorname{Re}(\alpha), \\
& g=g_{1}+g_{2}, \grave{g}=g_{1}-g_{2}, \\
& x_{1}=\sqrt{\left(y_{1}^{2}+\Delta_{1}^{2}\right)\left(y_{2}^{2}+\Delta_{2}^{2}\right),} \\
& x_{2}=\Delta_{1}^{2}+\Delta_{2}^{2}+2\left[\operatorname{Re}(\alpha)^{2}\left(g^{2}+\grave{g}^{2}\right)\right] \\
& x_{3}=\sqrt{x_{2}+(-1)^{\frac{k(k+1)}{2}} 2 x_{1}}, \\
& \zeta_{1}=\left[(-1)^{k} 2 g \operatorname{Re}(\alpha)+x_{3}\right] \text { and } \\
& \zeta_{2}=\left[y_{2} \Delta_{1}^{2}+y_{1} \Delta_{2}^{2}+(-1)^{\frac{k(k+1)}{2}} 2 g \operatorname{Re}(\alpha) x_{1}\right]
\end{aligned}
$$

However, from above system (4), one can note that $\left|\grave{\Psi}_{i}\right\rangle=\left\{K_{1}|+,+, \alpha\rangle, K_{2}|+,-, \alpha\rangle, K_{3}|-,+, \alpha\rangle\right.$, $\left.K_{4}|-,-, \alpha\rangle\right\}$, with $|\alpha\rangle$ is the coherent state and $K_{j}=\sum_{k=1}^{4} \exp \left(-i \lambda_{k} t\right) Q_{j k}$. Then we obtain $|\Psi\rangle$ as follows $|\Psi\rangle=U|\grave{\Psi}\rangle$.

The density matrix operator of the whole system, $\hat{\rho}(t)$, can be written as $\hat{\rho}(t)=|\Psi\rangle|\Psi\rangle$. Hence, the density operator of the two qubits, $\hat{\rho}_{R}(t)$, is given by:

$$
\hat{\rho}_{R}(t)=\operatorname{Tr}_{F}(\hat{\rho})=\frac{1}{4} V V^{\dagger},
$$

with $V=\left(\begin{array}{l}K_{1}+K_{2}+K_{3}+K_{4} \\ K_{1}-K_{2}+K_{3}-K_{4} \\ K_{1}+K_{2}-K_{3}-K_{4} \\ K_{1}-K_{2}-K_{3}+K_{4}\end{array}\right)$.

In Fig. (1), we investigate the effect of the qubitphoton coupling constants for the two qubits ( $g_{1}$ and $g_{2}$ ) on the atomic occupation probabilities $\rho_{11}, \rho_{22}, \rho_{33}$ and $\rho_{44}$. The occupation probabilities $\rho_{11}, \rho_{22}, \rho_{33}$ and $\rho_{44}$ have regular and periodic oscillations. We show that when the qubit-photon coupling constants for the two qubits are equal $\left(g_{1}=g_{2}, \grave{g}=0\right)$, the occupation probability $\rho_{11}$ starts from its minimum value, $\rho_{11}=0$, and it has maximum value at $\rho_{11}=0.75$. While $g_{1}=3 g_{2}, \grave{g}=1$, the occupation probability $\rho_{11}$ starts from the value, $\rho_{11}=0.3$, and it has maximum value at $\rho_{11}=0.5$. Also, when $g_{1}=7 g_{2}, \grave{g}=1.5$, the occupation probability $\rho_{11}$ starts from the value, $\rho_{11}=0.35$, and it has maximum value at $\rho_{11}=0.5$, and the number of periodic oscillations increases. When the qubit-photon coupling constants for the two qubits are equal $\left(g_{1}=g_{2}, \grave{g}=0\right)$, the occupation probability $\rho_{44}$ starts from its maximum value, $\rho_{44}=0.7$, and it has minimum value at $\rho_{44}=0$. While $g_{1}=3 g_{2}, \grave{g}=1$, the occupation probability $\rho_{44}$ starts from the value, $\rho_{44}=0.3$,and it has maximum value at $\rho_{44}=0.5$. Also, when $g_{1}=7 g_{2}, \grave{g}=1.5$, the occupation probability $\rho_{44}$ starts from the value, $\rho_{44}=0.35$, and it has maximum value at $\rho_{44}=0.5$, and the number of periodic oscillations increases. So, we see that the second and the third cases of $\rho_{11}$ and $\rho_{44}$ are the same. When the qubit-photon coupling constants for the two qubits are equal $\left(g_{1}=g_{2}, \grave{g}=0\right)$, the occupation probabilities $\rho_{22}$ and $\rho_{33}$ start from minimum value, $\rho_{22}=\rho_{33}=0.1$, and they have small oscillations. While $g_{1}=3 g_{2}$, $\grave{g}=1$, the occupation probabilities $\rho_{22}$ and $\rho_{33}$ start from the value, $\rho_{22}=\rho_{33}=0.15$, and the oscillations increase. Also, when $g_{1}=7 g_{2}, \grave{g}=1.5$, the occupation probabilities $\rho_{22}$ and $\rho_{33}$ start from the value, $\rho_{22}=\rho_{33}=0.2$, and the oscillations increase.

In Fig. (2), we investigate the effect of the detuning parameters $\left(\Delta_{1}\right.$ and $\left.\Delta_{2}\right)$ on the atomic occupation probabilities $\rho_{11}, \rho_{22}, \rho_{33}$ and $\rho_{44}$. The occupation probabilities $\rho_{11}, \rho_{22}, \rho_{33}$ and $\rho_{44}$ have regular and periodic oscillations. We observe, when $\Delta_{1}=0.5$, $\Delta_{2}=0$, the oscillations of the occupation probabilities to a great extent look like the case of the spontaneous emission. The occupation probability $\rho_{11}$ starts from its minimum value, $\rho_{11}=0$, and increases until it 

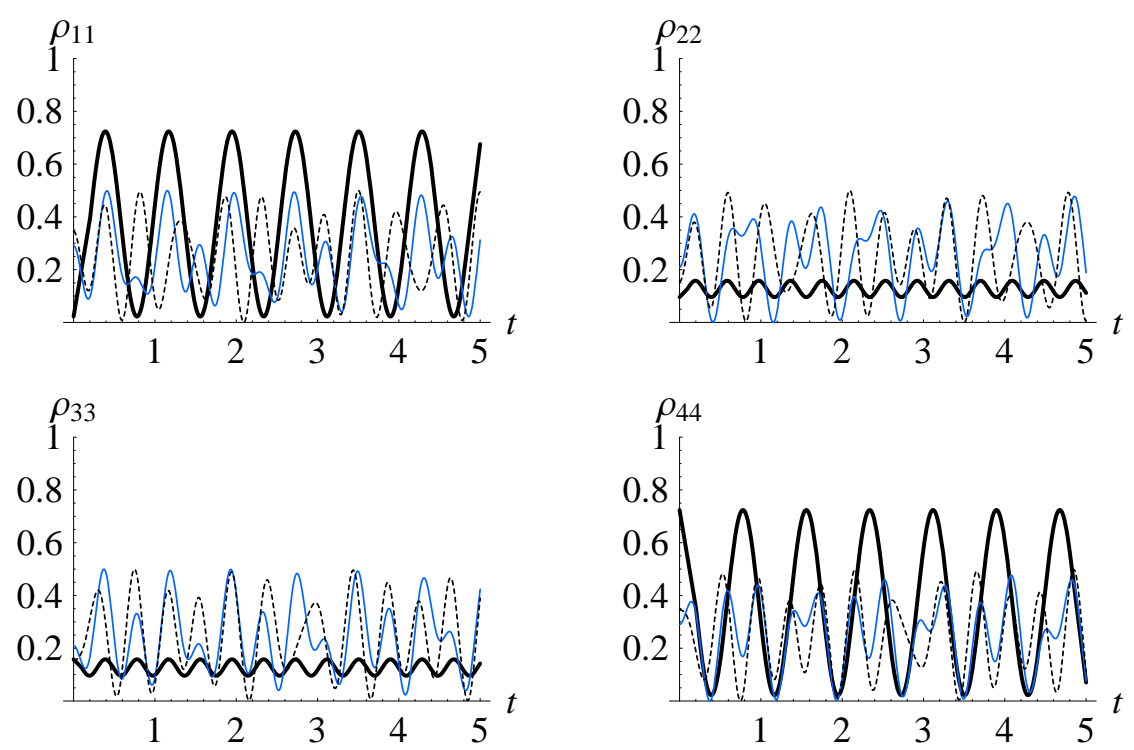

Figure 1: These figures show the the cases in which $\omega=1, \Delta_{1}=\Delta_{2}=0.5, g=2$, where solid, blue and dot curves correspond, respectively, to $\grave{g}=0\left(g_{1}=g_{2}\right), \grave{g}=1 g_{1}=3 g_{2}$ and $\grave{g}=1.5\left(g_{1}=7 g_{2}\right)$.

reaches maximum value at $\rho_{11}=1$ then decreases and increases gradually until it reaches its minimum value, $\rho_{11}=0$ (sudden death) and increases again and so on. While $\left(\Delta_{1}=0.5, \Delta_{2}=0.5\right)$, the occupation probability $\rho_{11}$ starts from the value, $\rho_{11}=0.3$, and it has maximum value at $\rho_{11}=0.5$. When $\left(\Delta_{1}=0.5, \Delta_{2}=0.8\right)$, the occupation probability $\rho_{11}$ starts from the value, $\rho_{11}=0.35$, and it has maximum value at $\rho_{11}=0.9$, and the number of periodic oscillations increase. The occupation probability $\rho_{44}$ starts from its maximum value, $\rho_{11}=1$, and decreases until it reaches minimum value at $\rho_{11}=0$, then increases and decreases gradually until it reaches its minimum value, $\rho_{11}=0$ (sudden death) and increases again and so on. While $\left(\Delta_{1}=0.5\right.$, $\left.\Delta_{2}=0.5\right)$, the occupation probability $\rho_{44}$ starts from the value, $\rho_{11}=0.3$, and it has maximum value at $\rho_{44}=0.5$. When $\left(\Delta_{1}=0.5, \Delta_{2}=0.8\right)$, the occupation probability $\rho_{44}$ starts from the value, $\rho_{44}=0.35$, and it has maximum value at $\rho_{44}=0.9$, and the number of periodic oscillations increases. The occupation probabilities $\rho_{22}$ and $\rho_{33}$ start from its minimum value, $\rho_{22}=\rho_{33}=0$, and increase then decrease and increase gradually until they reach maximum value at $\rho_{22}=\rho_{33}=1$ then decrease and increase gradually until it reaches the minimum value, $\rho_{22}=\rho_{33}=0$ (sudden death) and increase again and so on. When $\left(\Delta_{1}=0.5, \Delta_{2}=0.5\right)$, the occupation probabilities $\rho_{22}$ and $\rho_{33}$ start from the value, $\rho_{22}=\rho_{33}=0.3$, and they have maximum value at $\rho_{22}=\rho_{33}=0.5$. When $\left(\Delta_{1}=0.5, \Delta_{2}=0.8\right)$, the occupation probabilities $\rho_{22}$ and $\rho_{33}$ start from the value, $\rho_{22}=\rho_{33}=0.35$, and they have maximum value at $\rho_{22}=\rho_{33}=0.9$, and the number of periodic oscillations increase. We observe, the case of $\left(\Delta_{1}=0.5, \Delta_{2}=0\right)$ to a great extent look like the case of $\left(\Delta_{1}=0.5, \Delta_{2}=0.8\right)$. But the case of $\left(\Delta_{1}=0.5, \Delta_{2}=0.5\right)$ is different from the two former cases, where the difference between $\Delta_{1}$ and $\Delta_{2}$ is equal zero.

\section{The Information ENTROPIES}

The probability distribution for $N$ possible outcomes of measurements for an arbitrary quantum state of an operator $\sigma_{\gamma}$ is

$$
P\left(\sigma_{\gamma}\right)=\left\langle\Psi_{\gamma i}|\rho| \Psi_{\gamma i}\right\rangle,
$$

where $\left|\Psi_{\gamma i}\right\rangle$ eigenvector of the operator $\sigma_{\gamma}$ :

$$
\sigma_{\gamma}\left|\Psi_{\gamma i}\right\rangle=\eta_{\gamma i}\left|\Psi_{\gamma i}\right\rangle, \gamma=x, y, z, i=1,2, \ldots, N
$$

For a two two-level atoms, $N=4$ and by using the atomic reduced density operator $\rho_{R}(t)$, we obtain the information entropies of the atomic operators $\sigma_{x}, \sigma_{y}$ and $\sigma_{z}$ in the form [33,34]

$$
H\left(\sigma_{\gamma}\right)=-\sum_{i=1}^{4} P_{i}\left(\sigma_{\gamma}\right) \ln P_{i}\left(\sigma_{\gamma}\right), \gamma=x, y, z
$$

In Fig. (3), we investigate the effect of the qubitphoton coupling constants for the two qubits $\left(g_{1}\right.$ and $g_{2}$ ) on the information entropies $H\left(\sigma_{Z}\right), H\left(\sigma_{Y}\right), H\left(\sigma_{X}\right)$ 

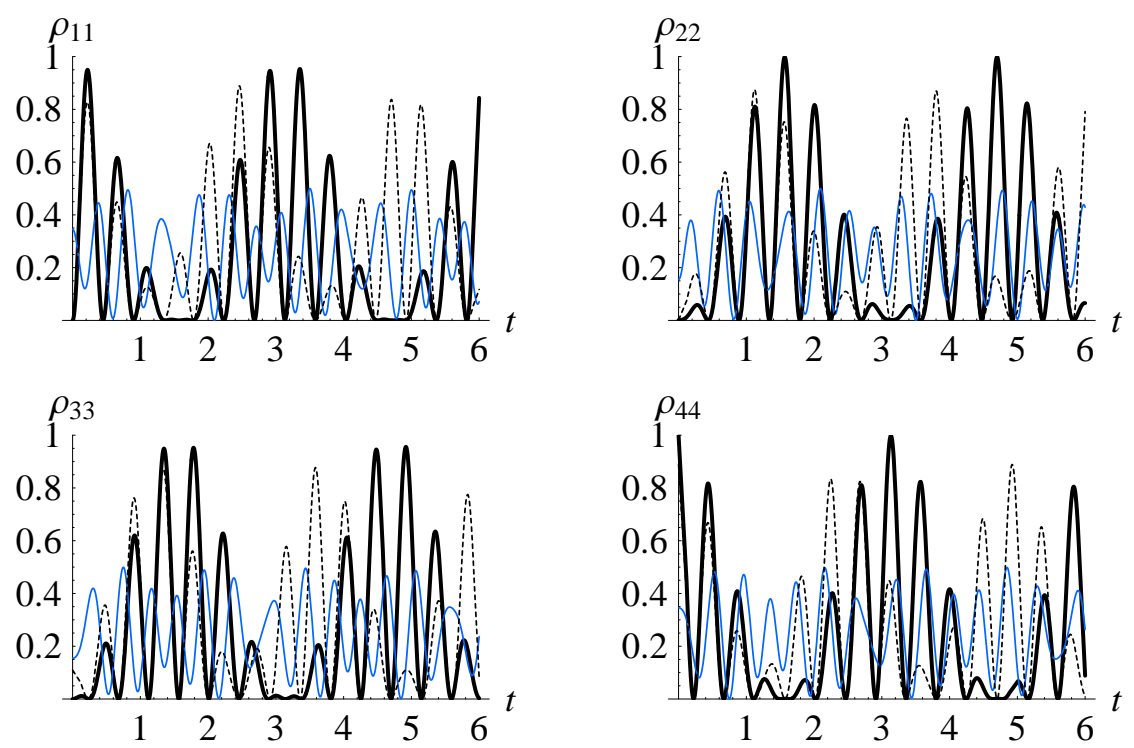

Figure 2: These figures present the cases in which $\omega=1, g=2, \grave{g}=1.5\left(g_{1}=7 g_{2}\right), \Delta_{1}=0.5$, where solid, blue and dot curves correspond respectively to, $\Delta_{2}=0, \Delta_{2}=0.5$ and $\Delta_{2}=0.8$.

and the Von Neumann entropy $S\left(\rho_{A}\right)$. The information entropies $H\left(\sigma_{Z}\right), H\left(\sigma_{Y}\right)$ and $H\left(\sigma_{X}\right)$ have regular and periodic oscillations. We show that when the qubit-photon coupling constants for the two qubits are equal $\left(g_{1}=g_{2}, \grave{g}=0\right)$, the information entropy $H\left(\sigma_{Z}\right)$ starts from its minimum value, $H\left(\sigma_{Z}\right)=0.85$, and increases until it reaches its maximum value at $H\left(\sigma_{Z}\right)=1.25$. While $g_{1}=3 g_{2}, \grave{g}=1$, the information entropy $H\left(\sigma_{Z}\right)$ starts from its maximum value, $H\left(\sigma_{Z}\right)=1.38$, and it has minimum value at $H\left(\sigma_{Z}\right)=$ 0.75. When $g_{1}=7 g_{2}, \grave{g}=1.5$, the information entropy $H\left(\sigma_{Z}\right)$ starts from the value, $H\left(\sigma_{Z}\right)=1.33$, and it has minimum value at $H\left(\sigma_{Z}\right)=0.95$, and the number of periodic oscillations decreases. When the qubitphoton coupling constants for the two qubits are equal $\left(g_{1}=g_{2}, \grave{g}=0\right)$, the information entropy $H\left(\sigma_{X}\right)$ starts from its minimum value, $H\left(\sigma_{X}\right)=1.15$, and it has maximum value at $H\left(\sigma_{X}\right)=1.35$. While $g_{1}=3 g_{2}$, $\grave{g}=1$, the information entropy $H\left(\sigma_{X}\right)$ has small oscillations. Also, when $g_{1}=7 g_{2}, \grave{g}=1.5$, the oscillations of the information entropy $H\left(\sigma_{X}\right)$ decreases remarkably. When the qubit-photon coupling constants for the two qubits are equal $\left(g_{1}=g_{2}, \grave{g}=0\right)$, the information entropy $H\left(\sigma_{Y}\right)$ starts from its maximum value, $H\left(\sigma_{Y}\right)=1.35$, and it decreases until it reaches its minimum value at $H\left(\sigma_{Y}\right)=0.5$. While $g_{1}=3 g_{2}, \grave{g}=1$, the information entropy $H\left(\sigma_{y}\right)$ has small oscillations. Also, when $g_{1}=7 g_{2}, \grave{g}=1.5$, the oscillations of the information entropy $H\left(\sigma_{Y}\right)$ decrease. When the qubitphoton coupling constants for the two qubits are equal $\left(g_{1}=g_{2}, \grave{g}=0\right)$, the Von Neumann entropy $S\left(\rho_{A}\right)$ starts from its minimum value, $S\left(\rho_{A}\right)=0.25$, and it has maximum value at $S\left(\rho_{A}\right)=0.45$. The oscillations of the Von Neumann entropy $S\left(\rho_{A}\right)$ has small phase. While $g_{1}=3 g_{2}, \grave{g}=1$, and $\left(g_{1}=7 g_{2}, \grave{g}=1.5\right)$, the phase and the number of the oscillations of the Von Neumann entropy $S\left(\rho_{A}\right)$ increases remarkably. Also, the maximum and minimum values of the Von Neumann entropy $S\left(\rho_{A}\right)$ increase remarkably.

In Fig. (4), we investigate the effect of the detuning parameters $\left(\Delta_{1}\right.$ and $\left.\Delta_{2}\right)$ on the information entropies $H\left(\sigma_{Z}\right), H\left(\sigma_{Y}\right), H\left(\sigma_{X}\right)$ and the Von Neumann entropy $S\left(\rho_{A}\right)$. The information entropies $H\left(\sigma_{Z}\right), H\left(\sigma_{Y}\right)$ and $H\left(\sigma_{X}\right)$ have regular and periodic oscillations. We observe, when $\Delta_{1}=0.5, \Delta_{2}=0$, and $\left(\Delta_{1}=0.5, \Delta_{2}=0.8\right)$, the information entropy $H\left(\sigma_{Z}\right)$ has many oscillations. While, when $\left(\Delta_{1}=0.5, \Delta_{2}=0.5\right)$, the information entropy $H\left(\sigma_{Z}\right)$ has few number of oscillations. When $\Delta_{1}=0.5, \Delta_{2}=0$, and $\left(\Delta_{1}=0.5, \Delta_{2}=0.5\right)$, the information entropy $H\left(\sigma_{X}\right)$ has many oscillations. While, when $\left(\Delta_{1}=0.5, \Delta_{2}=0.8\right)$, the information entropy $H\left(\sigma_{X}\right)$ reaches fixed value at $H\left(\sigma_{X}\right)=1.3$. When $\Delta_{1}=0.5, \Delta_{2}=0$, and $\left(\Delta_{1}=0.5, \Delta_{2}=0.8\right)$, the information entropy $H\left(\sigma_{Y}\right)$ has the same maximum and minimum value and $H\left(\sigma_{Y}\right)$ starts from $H\left(\sigma_{Y}\right)=1.2$. While, when $\Delta_{1}=0.5, \Delta_{2}=0.5$, the information entropy $H\left(\sigma_{Y}\right)$ starts from $H\left(\sigma_{Y}\right)=1.4$ and has small phase of the oscillations. When $\Delta_{1}=0.5, \Delta_{2}=0$, and $\left(\Delta_{1}=0.5, \Delta_{2}=0.8\right)$, the Von Neumann entropy $S\left(\rho_{A}\right)$ starts from its minimum value $S\left(\rho_{A}\right)=0$ and has regular and periodic oscillations. While, when $\Delta_{1}=0.5$, $\Delta_{2}=0.5$, the Von Neumann entropy $S\left(\rho_{A}\right)$ starts from 

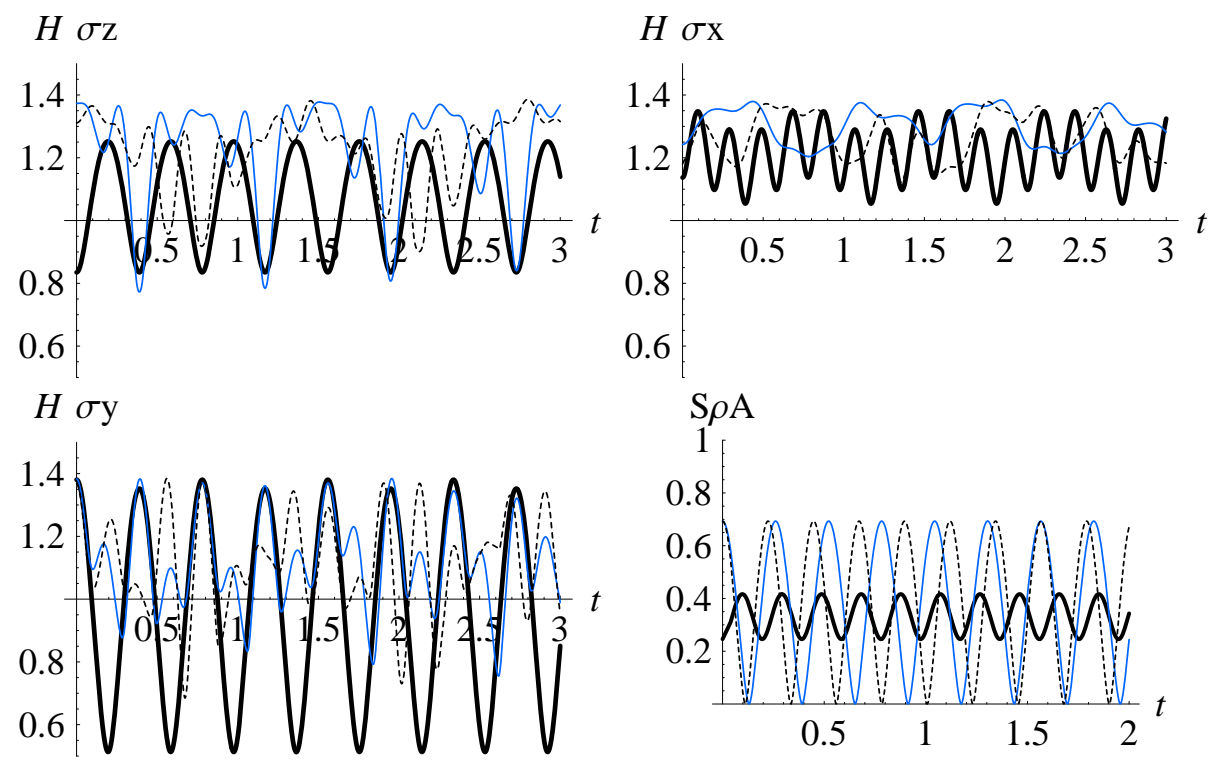

Figure 3: These figures present the cases in which $\omega=1, \Delta_{1}=\Delta_{2}=0.5, g=2$, where solid, blue and dot curves correspond, respectively, to $\grave{g}=0\left(g_{1}=g_{2}\right), \grave{g}=1\left(g_{1}=3 g_{2}\right)$ and $\grave{g}=1.5\left(g_{1}=7 g_{2}\right)$

its maximum value $S\left(\rho_{A}\right)=0.7$ and and also has regular and periodic oscillations.

\section{CONCLUSION}

In this paper, we analytically proposed an exact solution for two-qubit Rabi model in the general case. We studied the atomic occupation probabilities $\rho_{11}$, $\rho_{22}, \rho_{33}$ and $\rho_{44}$, also, the information entropies $H\left(\sigma_{Z}\right)$, $H\left(\sigma_{Y}\right), H\left(\sigma_{X}\right)$ and the Von Neumann entropy $S\left(\rho_{A}\right)$ for some special values of the qubit-photon coupling constants for the two qubits ( $g_{1}$ and $\left.g_{2}\right)$ and the detuning parameters $\left(\Delta_{1}\right.$ and $\left.\Delta_{2}\right)$. When the qubit-photon coupling constant $g_{2}$ increases the phase of the oscillations of the atomic occupation probabilities $\rho_{11}, \rho_{44}$ and the information entropies $H\left(\sigma_{Y}\right)$ decreases, but the phase of the oscillations of the atomic occupation probabilities $\rho_{22}, \rho_{33}$ and the Von Neumann entropy $S\left(\rho_{A}\right)$ increases. The occupation probabilities $\rho_{11}, \rho_{22}$, $\rho_{33}$ and $\rho_{44}$, also, the information entropies $H\left(\sigma_{Z}\right)$, $H\left(\sigma_{Y}\right), H\left(\sigma_{X}\right)$ and the Von Neumann entropy $S\left(\rho_{A}\right)$ have regular and periodic oscillations. When $\Delta_{1}=0.5$, $\Delta_{2}=0$, the oscillations of the occupation probabilities to a great extent look like the case of the spontaneous emission. The occupation probability $\rho_{11}$ reaches the case of sudden death.

\section{REFERENCES}

[1] I. Rabi, Phys. Rev. 49 (1936) 324.
[2] M. O. Scully and M. S. Zubairy, Quantum optics (Cambridge UniversityPress, Cambridge), (1997).

[3] M. Wagner, Unitary Transformations in Solid State Physics (North-Holland, Amsterdam), (1986).

[4] J. .M. Raimond, M. Brune and S. Haroche, Rev. Mod. Phys. 73(2001) 565.

[5] D. Liebfried, R. Blatt, C. Monroe and D. Wineland, Rev. Mod. Phys. 75 (2003) 281.

[6] D. Englund, A. Faraon, I. Fushman, N. Stoltz and J. Vučković, Nature 450 (2007) 857.

[7] Y. Nakamura, Y. A.Pashkin, and Tsai J S, Phys. Rev. Lett. 87 (2001) 246601.

[8] A. Wallraff, Schuster D I, Blais A, Frunzio L, Huang R S, Majer J, Kumar S, Girvin S M and Schoelkopf R J, Nature 431 (2004) 162.

[9] T. Niemczyk, Deppe F, H. Huebl, E. P. Menzel, F. Hocke, M. J. Schwarz, J. J. Garcia-Ripoll, D. Zueco, T. H ummer, E. Solano, A. Marx and R. Gross, $\mathrm{Na}$ ture Phys. 6 (2010) 772.

[10] P. Forn-Díaz, J. Lisenfeld, D. Marcos, J. J. GarcíaRipoll, E. Solano, C. J. P. M. Harmans, and J. E. Mooij, Phys. Rev. Lett. 105 (2010) 237001.

[11] I. Chiorescu, P. Bertet, K. Semba, Y. Nakamura, C. J. P. M. Harmans and J. E.Mooij, Nature 431 (2004) 159. 

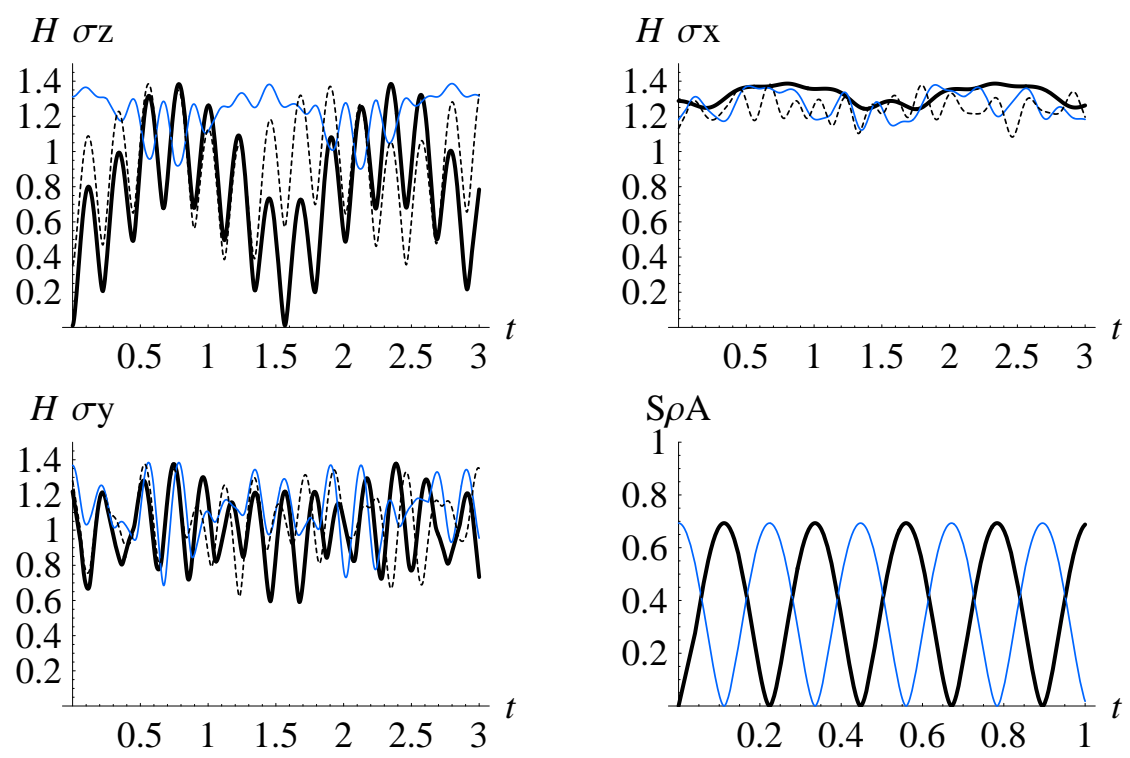

Figure 4: These figures present the case in which $g=2, \grave{g}=1.5\left(g_{1}=7 g_{2}\right), \Delta_{1}=0.5$, where solid, blue and dot curves correspond, respectively, to $\Delta_{2}=0, \Delta_{2}=0.5$ and $\Delta_{2}=0.8$.

[12] A. Fedorov, A. K. Feofanov, P. Macha, P. FornDíaz, C. J. P. M. Harmans and J. E. Mooij, Phys. Rev. Lett. 105 (2010) 060503.

[13] S. Longhi, Opt. Lett. 36 (2011) 3407.

[14] A. Crespi, S. Longhiand R. Osellame, Phys. Rev. Lett. 108 (2012) 163601.

[15] G. A. Prinz, Science 282 (1998) 1660.

[16] S. A. Wolf,et al., Science 294 (2001) 1488.

[17] R. Winkler, Phys. Rev. B 62 (2000) 4254.

[18] X. F. Wang, P. Vasilopoulos and F. M. Peeters, Appl. Phys. Lett. 80 (2002) 1400.

[19] S. Bandyopadhyay, Phys. Rev. B 61 (2000) 13813.

[20] FG - R. L. Fulton and M. Gouterman, J. Chem. Phys. 35 (1961) 1059.

[21] J. Peng, Z. Ren, Daniel Braak, Guangjie Guo, Guoxing Ju, Xin Zhang and Xiaoyong Guo, J. Phys. A: Math. Theor. 47 (2014) 265303.

[22] D. Braak, Continued fractions and the Rabi model, J. Phys. A: Math. Theor. 46 (2013) 175301.

[23] H. Zhong, Q. Xie, and C. Lee, Analytical eigenstates for the quantum Rabi model, arXiv:1305.6782v1 [quant-ph] 29 May (2013).
[24] L. Duan, S. He, and Q. Chen, Concise analytic solutions to the quantum Rabi model with two arbitrary qubits, arXiv:1405.0555v2 [quant-ph] 14 Mar (2015).

[25] D. Braak, Note on the Analytical Solution of the Rabi Model, arXiv:1210.4946v2 [math-ph] 23 Nov (2012).

[26] J. Peng, Z. Ren, Haitao Yang, Guangjie Guo, Xin Zhang, Guoxing Ju, Xiaoyong Guo, Chaosheng Deng, Guolin Hao, Algebraic structure of the twoqubit quantum Rabi model and its solvability using Bogoliubov operators, arXiv:1501.06637v1 [quant-ph] 27 Jan (2015).

[27] Q. -H. Chen, L. Duan, and S. He, Analytically exact solutions to the quantum Rabi model with two different qubits: Extended coherent states approaches, arXiv:1405.0555v1 [quant-ph] 3 May (2014).

[28] J. Peng, Z. Ren, Guangjie Guo, Guoxing Ju, and Xiaoyong Guo, Eur. Phys. J. D 67 (2013) 162.

[29] F. Altintas, Dissipative dynamics of atom-field entanglement in the ultrastrong-coupling regime, arXiv:1305.1110v2 [quant-ph] 29 Oct (2013).

[30] E. T. Jaynes and F. W. Cummings, Proc. IEEE 51 (1963) 89.

[31] W. W. Deng and Gao-Xiang Li, Eur. Phys. J. D 67 (2013) 91. 
[32] J. Peng, Zhongzhou Ren, Daniel Braak, Guangjie Guo, Guoxing Ju, Xin Zhang and Xiaoyong Guo, J Phys. A Math. Theor. 47 (2014) 265303.

[33] A.-S.F. Obada, S. Abdel-Khalek, M.M.A. Ahmed and D.A.M. Abo-Kahla, Optics Communications 282
(2009) 914.

[34] J. Sanchez-Ruiz, Phys. Lett. A 201 (1995) 125; J. Sanchez-Ruiz, Phys. Lett. A 244 (1998) 189; T.M.ElShahat, S.Abdel-Khalek, M.Abdel-Aty and A-S F.Obada, Chaos Solitons and Fractals 18 (2003) 289. 